\title{
Tremor suppression for 4-DOFs biodynamic hand model using genetic algorithm
}

\begin{abstract}
A person who has severe hand tremor will have difficulty in doing specific tasks such as eating, combing or holding any objects. Currently, there is no medication that can cure the tremor. Thus, this study proposes the active tremor control, in which an intelligent controller is applied to suppress the hand tremor. The main objective is to optimise the proportionalintegral (PI) controller using genetic algorithm (GA). A linear voice coil actuator (LVCA) is applied onto a four degree of freedom (4-DOF) human hand model represented in state space. The findings of the study demonstrate that the PI controller optimised by GA gives excellent performance in reducing the tremor error. Based on the frequency evaluation, the PI controller performance was roughly around $84 \%$ in reducing the peak of simulated hand tremor. The outcomes provide an important contribution towards achieving novel methods in suppressing hand tremor model by means of intelligent control.
\end{abstract}

Keyword: Human hand tremor; Active tremor control; Genetic algorithm 